\title{
Spin-Selective Kondo Insulator: Cooperation of Ferromagnetism and the Kondo Effect
}

\section{$\operatorname{AUTHOR}(S)$ :}

Peters, Robert; Kawakami, Norio; Pruschke, Thomas

\section{CITATION:}

Peters, Robert ... [et al]. Spin-Selective Kondo Insulator: Cooperation of Ferromagnetism and the Kondo Effect. Physical Review Letters 2012, 108(8): 086402.

\author{
ISSUE DATE: \\ 2012-02 \\ URL: \\ http://hdl.handle.net/2433/154856 \\ RIGHT: \\ (C) 2012 American Physical Society.
}




\title{
Spin-Selective Kondo Insulator: Cooperation of Ferromagnetism and the Kondo Effect
}

\author{
Robert Peters* and Norio Kawakami \\ Department of Physics, Kyoto University, Kyoto 606-8502, Japan \\ Thomas Pruschke \\ Department of Physics, University of Göttingen, 37077 Göttingen, Germany
}

(Received 26 October 2011; published 22 February 2012)

\begin{abstract}
We propose the notion of a spin-selective Kondo insulator, which provides a fundamental mechanism to describe the ferromagnetic phase of the Kondo lattice model with antiferromagnetic coupling. This unveils a remarkable feature of the ferromagnetic metallic phase: the majority-spin conduction electrons show metallic while the minority-spin electrons show insulating behavior. The resulting Kondo gap in the minority-spin sector, which is due to the cooperation of ferromagnetism and partial Kondo screening, evidences a dynamically induced commensurability for a combination of minority-spin electrons and parts of localized spins. Furthermore, this mechanism predicts a nontrivial relation between the macroscopic quantities such as electron magnetization, spin polarization, and electron filling.
\end{abstract}

DOI: 10.1103/PhysRevLett.108.086402

Even 30 years after their discovery heavy-fermion systems attract much attention due to their fascinating properties. Apart from being Fermi liquids with an effective mass thousands of times as large as the free electron one, they show all kinds of competing or coexisting phases, and at the boundaries between these phases one frequently observes quantum phase transitions, accompanied by barely understood non-Fermi liquid behavior [1-3]. Heavy-fermion compounds usually include lanthanides or actinides with open $4 f$ or $5 f$ shells, which in the simplest theoretical modeling can be viewed as a regular lattice of local moments coupled to the conduction electrons. This coupling typically leads to two competing mechanisms: the long-ranged RKKY interaction and the local Kondo screening. While the RKKY interaction favors a magnetically ordered state, the Kondo screening is usually considered to form a paramagnetic heavy-fermion state. The competition of these two mechanisms can be easily understood in terms of the Doniach phase diagram [4].

While in most heavy-fermion compounds the magnetic order is antiferromagnetic, there is a certain class of compounds showing ferromagnetic order. For example, the recently discovered $\mathrm{YbNi}_{4} \mathrm{P}_{2}$ is a ferromagnetically ordered heavy-fermion compound which seems to be very close to a quantum critical point [5]. Taking such ferromagnetic heavy-fermion compounds as motivation, we analyze in detail the mechanism stabilizing the ferromagnetic state. An interesting question in this context is, if and how the Kondo effect accounts for the ferromagnetic state [6-9].

In this Letter, we propose a spin-selective Kondo insulator, where the Kondo screening plays an essential role in stabilizing the ferromagnetic metallic state at zero temperature, which elucidates a previously unrecognized
PACS numbers: 71.10.Fd, 71.27.+a, 71.30.+h, 75.20.Hr feature of the ferromagnetic phase: the majority-spin (minority-spin) conduction electrons are in a metallic (insulating) state. We claim that this notion is not specific to certain choices of system parameters but is fundamental and ubiquitous for the ferromagnetic phase in the Kondo lattice model. Because of partial Kondo screening, parts of the local moments are bound to the electrons, resulting in a dynamically induced commensurability which is essential for producing the gap in the minority-spin electrons. We find that this commensurability condition leads to a nontrivial relation between electron magnetization, spin polarization, and electron filling.

The competition or cooperation between the magnetic phase mediated by the RKKY interaction and the Kondo screening can be modeled via a Kondo lattice model with antiferromagnetic coupling between the local moments and the conduction electrons. The Kondo lattice model reads $[4,10,11]$,

$$
H=t \sum_{\langle i, j\rangle \sigma} c_{i \sigma}^{\dagger} c_{j \sigma}+J \sum_{i} \vec{S}_{i} \vec{s}_{i}, \quad \vec{s}_{i}=c_{i \sigma_{m}}^{\dagger} \vec{\rho}_{\sigma_{m} \sigma_{n}} c_{i \sigma_{n}},
$$

where $c_{i \sigma}^{\dagger}$ creates an electron on site $i$ with spin direction $\sigma, \vec{\rho}$ represents the vector of Pauli matrices, and $\vec{S}_{i}$ represents the local spins which are coupled to the electrons via an antiferromagnetic spin-spin interaction with strength $J>0$.

To solve the Kondo lattice model we use the dynamical mean field theory (DMFT) [12-14]. DMFT maps the lattice model onto a quantum impurity model with a fermionic bath being determined self-consistently. Although being an approximation to real systems, DMFT has provided many insights into the physical properties and can even capture subtle differences in the lattice geometry. For solving the impurity model, we use the numerical renormalization group (NRG) $[15,16]$, which is able to 
reliably calculate spectral functions at very low temperatures $[17,18]$.

First, we briefly summarize the known DMFT results for the Kondo lattice model $[10,11,19,20]$ (A discussion on the RKKY interaction within DMFT can be found in [19].) At half filling there is a pronounced antiferromagnetic Néel state for weak coupling, which vanishes with increasing coupling strength $J$ via a continuous transition to a paramagnetic insulating state, the Kondo insulator. Doping slightly away from half filling this transition changes into a transition between an antiferromagnetic state (possibly spin-density wave) and a paramagnetic metallic state. Especially the paramagnetic state around half filling is dominated by the Kondo effect, where the Kondo screening of localized spins results in a large Fermi surface accompanied by a narrow band and a gap close to the Fermi energy. Away from half filling the effects of Kondo screening become less important as there is an imbalance between local moments and available conduction electrons. Such a tendency might be even stronger when the system enters a ferromagnetic state realized at low fillings, because an additional imbalance between spin-up and spindown electrons arises. Contrary to this naive expectation, however, we demonstrate here that the Kondo screening plays an essential role even in the ferromagnetic phase. In particular, we reveal that the cooperation of ferromagnetism and Kondo screening can realize a novel kind of Kondo insulating state in the ferromagnetic metallic phase.

Figure 1 shows the local spin-resolved spectral functions calculated in the ferromagnetic phase for a Bethe lattice with antiferromagnetic Kondo coupling $J / W=0.25$ (bandwidth $W=4 t$ ). For this coupling strength the ferromagnetic phase extends from a nearly empty system to approximately $n^{c}=n_{\uparrow}^{c}+n_{\downarrow}^{c}=0.5$. One finds a striking difference in the spectral functions, which has not been

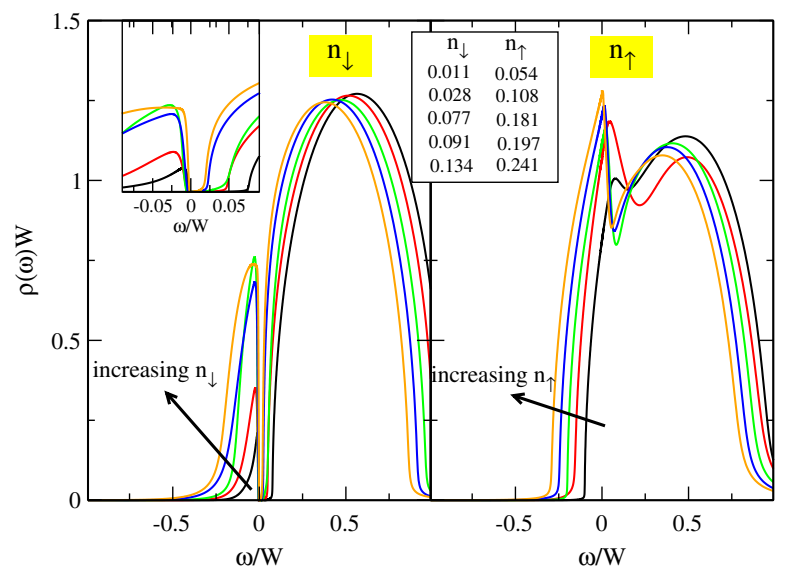

FIG. 1 (color online). Spin-resolved spectral functions for the ferromagnetic state in the Kondo lattice model $J / W=0.25$. The inset shows a magnification around the Fermi energy for the spin-down component illustrating the gap in the spectral function. recognized previously, for the majority spin $\left(n_{\uparrow}^{c}\right)$ and the minority spin $\left(n_{\downarrow}^{c}\right)$. While in the majority-spin spectral function a peak at the Fermi energy $\omega=0$ and a dip for $\omega>0$ can be found, there is a gap at the Fermi energy in the minority-spin spectral function. It is important to note that such a gap is not present in the ferromagnetic phase for a Kondo lattice model with ferromagnetically coupled spins. We propose that this gap in the spectral function is due to a partial Kondo screening of the localized spins, which results in an intriguing state: although the ferromagnetic state is metallic, only the majority-spin electrons contribute to the low-temperature properties, in particular, transport. The minority spins, even though not completely depleted, form an insulator, which we name spin-selective Kondo insulator.

Increasing the occupation number, the dip in the majority-spin spectral function moves closer to the Fermi energy and becomes more pronounced. Eventually, the ferromagnetic state is replaced by a paramagnetic state, for which the spectral functions for both spin components suffer from the typical suppression of the DOS for $\omega>0$ due to Kondo screening. Increasing the occupation towards half filling this dip becomes deeper and finally moves to the Fermi energy, forming the Kondo insulator.

Clear evidence showing that the above insulating gap is indeed caused by the Kondo screening can be found in the dependence of the gap width $\Delta$ on the occupation and coupling strength, shown in Fig. 2. The left panel in Fig. 2 displays the dependence of the gap width on the coupling strength. For this purpose the minority-spin occupancy was kept constant (also resulting in a nearly constant majority-spin occupancy). The dependency on $J$ perfectly obeys a Kondo-temperature-like form $\Delta \sim$ $\exp (-a / J)$ with a fitting constant $a$, suggesting that the Kondo physics is essential for the gap formation. As a function of increasing filling the gap width decreases
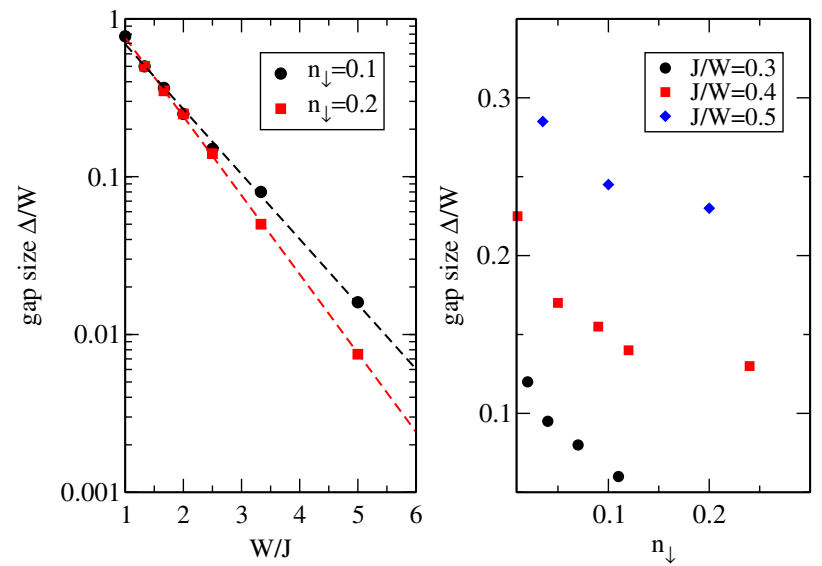

FIG. 2 (color online). Gap width $\Delta / W$ in the minority-spin spectral function depending on the spin-coupling $J$ and the occupation $n_{\downarrow}^{c}$. The temperature of the system is $T / W=$ $3 \times 10^{-4}$. The lines in the left panel are fits as $\sim \exp (-a / J)$. 
monotonically, as shown in the right panel of Fig. 2. As soon as the ferromagnetic phase vanishes, the gap at the Fermi energy closes, too, and the minority and majority spectral functions look similar to the right panel in Fig. 1.

Let us now elucidate the basic physics behind this ferromagnetic state. In Fig. 3 the magnetization of the conduction electrons $\langle m\rangle=n_{\uparrow}^{c}-n_{\downarrow}^{c}$ and the polarization of the localized spins $-\left\langle S_{z}\right\rangle$ is shown. Note that the local spin polarization always has the sign opposite to the conduction electron magnetization due to the antiferromagnetic coupling, thus, $-\left\langle S_{z}\right\rangle$ has the same sign. Increasing the number of conduction electrons, the magnetization of the electrons first increases due to increasing filling, and eventually decreases again due to the suppression of the ferromagnetic state. On the other hand, the spins are almost fully polarized for a nearly empty lattice, with monotonically decreasing polarization for increasing conduction electron number. In the spirit of a pseudofermion representation, let us assume that the localized spins are actually formed by a local half-filled and strongly interacting energy level so that $\left\langle S_{z}\right\rangle=\left(n_{\uparrow}^{f}-n_{\downarrow}^{f}\right) / 2$ and $n_{\uparrow}^{f}+n_{\downarrow}^{f}=1$

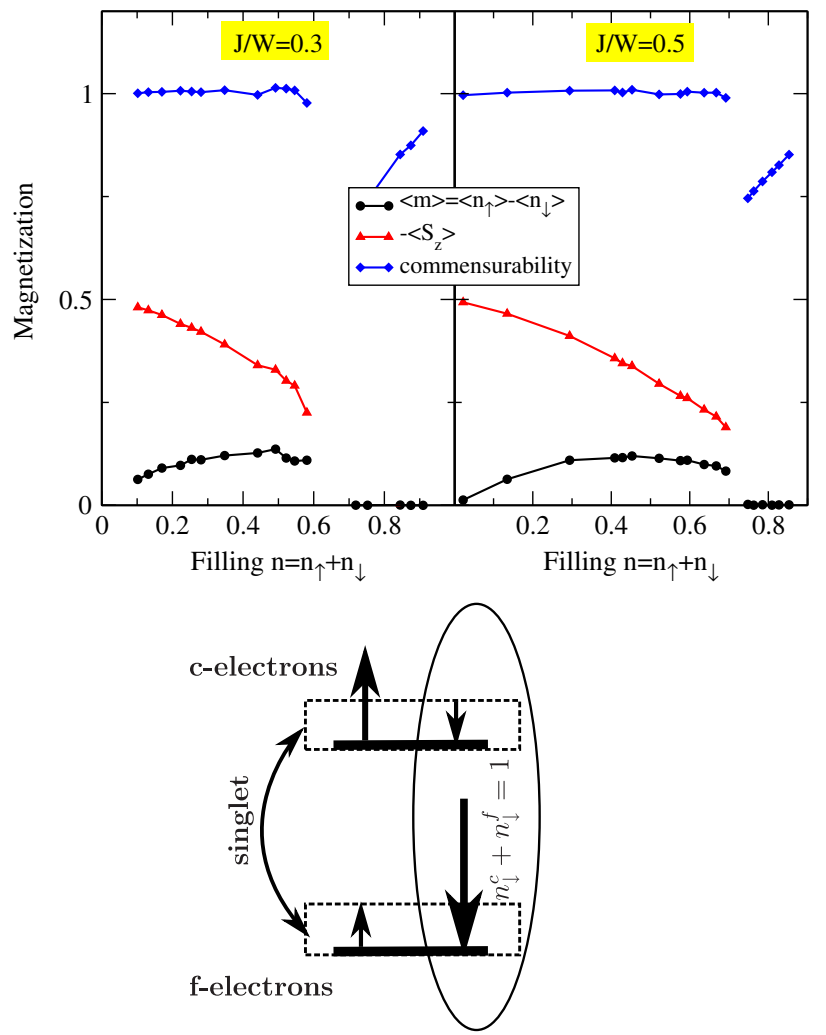

FIG. 3 (color online). Upper panel: Magnetization and "commensurability" $\left(n_{\downarrow}^{c}+n_{\downarrow}^{f}\right)$, for two different coupling strengths and different occupation numbers calculated for a Bethe lattice at $T / W=3 \times 10^{-4}$. The electron magnetization is shown as $\langle m\rangle=n_{\uparrow}^{c}-n_{\downarrow}^{c}$, while the spin expectation value $\left\langle S_{z}\right\rangle$ is shown mirrored as $-\langle S z\rangle$. The commensurability condition is explained in the text. Lower panel: sketch of the local configuration (see text). (defining $n_{\sigma}^{f}$ as the spin-dependent occupation of this level). Remarkably, we find that the following nontrivial commensurability condition holds within the ferromagnetic state:

$$
n_{\downarrow}^{c}+n_{\downarrow}^{f}=1,
$$

as can be seen in Fig. 3. Note that Eq. (1) is equivalent to $n_{\uparrow}^{f}=n_{\downarrow}^{c}$. It should be noticed that this condition is not a priori given but is generated dynamically due to manybody effects. To clarify the origin of the above commensurability we propose that a partial local Kondo singlet is formed in which $\left\langle n_{\downarrow}^{c}\right\rangle$ majority and minority electrons participate, thus combining all spin-down conduction electrons together with a part of the $f$ electrons and the spin-up conduction electrons to a Kondo spin singlet. Here, we have assumed that spin-down is the minorityspin direction. The remaining majority-spin conduction electrons and spin-down $f$ electrons form a ferromagnetic state (see a sketch in the lower panel of Fig. 3). That the number of spin-down electrons including $f$ and conduction electrons sums up to unity gives a commensurable situation, which results in a gap at the Fermi energy. On the other hand, for the majority spins there is not such a commensurability condition, but $n_{\uparrow}^{c}+n_{\uparrow}^{f}=n^{c}=n_{\uparrow}^{c}+n_{\downarrow}^{c}$ holds. Therefore, this partial Kondo screening results in an insulating state for the minority spin, while the majorityspin electrons remain metallic. For this reason we have called this state a "spin-selective Kondo insulator." The commensurability condition (1) smoothly connects to the Kondo insulator at half filling, suggesting that this ferromagnetic state should exist up to half filling. However, our results clearly show that there is a transition from this ferromagnetic phase to a paramagnetic state at electron fillings for $n^{c}>n_{\text {ferro }}$. This is only possible if the expectation value $\langle S z\rangle$ jumps, leading to a discontinuous phase transition at $n_{\text {ferro }}$. Our finding of the discontinuous transition completely agrees with the recent analytical results showing that nonanalytic terms prevent the continuous transition from a ferromagnet to a paramagnet [21].

A further important consequence deduced directly from the commensurability condition (1) is a nontrivial relation between electron magnetization, spin polarization, and occupation number:

$$
2\langle S z\rangle+\langle m\rangle=\left\langle n^{c}\right\rangle-1 .
$$

This formula connects these three quantities which are otherwise independent from each other. By arranging itself in this way the system can gain an additional energy originating from the partial Kondo screening. Note that it should be possible to verify such a relation experimentally.

The formation of the gap in the spectral function does not depend on the lattice geometry. For example, it can also be found in DMFT calculations for a two-dimensional square lattice. Figure 4 shows the momentum-resolved 

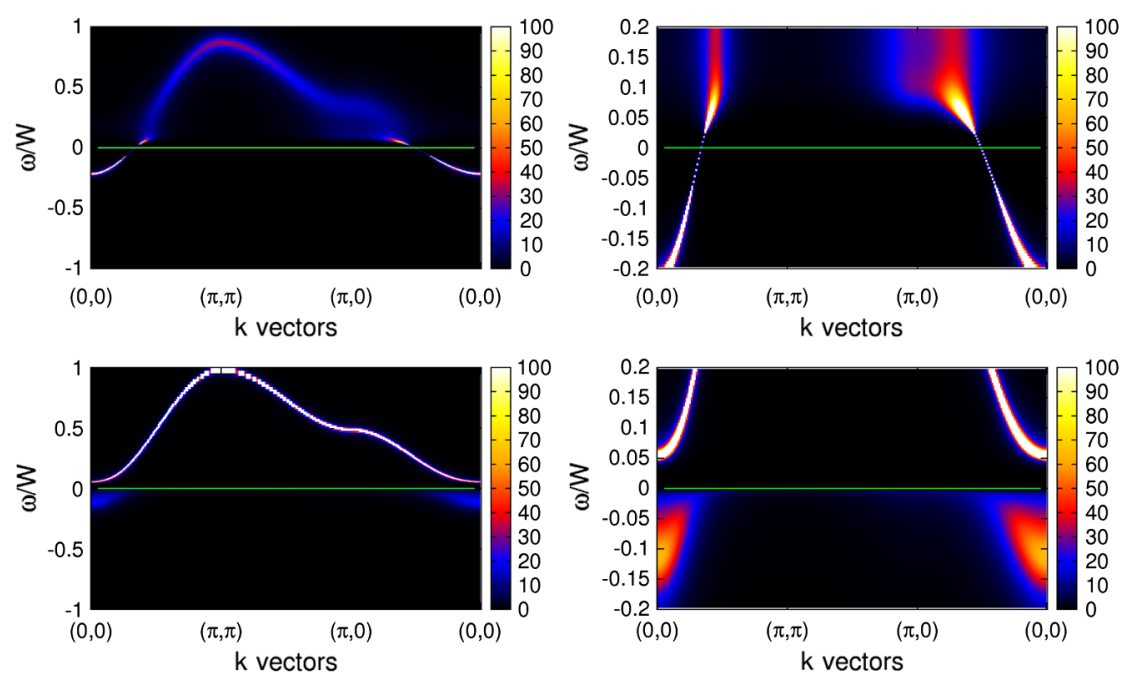

FIG. 4 (color online). Momentum-resolved spectral functions for the square lattice and $J / W=0.3, n=n_{\uparrow}^{c}+n_{\downarrow}^{c}=0.25, m=$ $n_{\uparrow}^{c}-n_{\downarrow}^{c}=0.1$. The right side always shows a magnification of the left side around the Fermi energy $\omega=0$ represented by the green line. From top to bottom the figures show the majority-spin and the minority-spin spectral function, respectively.

spectral functions for $J / W=0.3$ and $n_{\uparrow}^{c}+n_{\downarrow}^{c}=0.25$ for a square lattice. The right panels are magnifications around the Fermi energy. In the minority-spin spectral function (bottom right) the gap can be clearly seen. While the majority-spin electrons are renormalized for $\omega>0$ with a finite lifetime, the minority-spin electrons are renormalized for $\omega<0$. This behavior is also visualized in Fig. 5, in which the momentum-resolved occupation number is shown. While for the majority-spin electrons the occupation number distribution looks similar to the noninteracting case, for the minority-spin electrons this function is actually smeared out as compared to a reference noninteracting system; i.e., we indeed observe a large Fermi volume here.

Using DMRG, we have confirmed that the spin-selective Kondo insulator together with the commensurability condition can be found for the ferromagnetic phase of the one-dimensional (1D) Kondo lattice model, too. In fact, previous calculations for the ferromagnetic ground state observed a magnetization $S_{\text {tot }}=1 / 2\left(L-N_{c}\right)$ [22] ( $L$ system length, $N_{c}$ electron number), which supports the commensurability condition, and two separated bands in the spectral functions [23]. From these precise analyses of the 1D model, we conclude that our finding is ubiquitous for the Kondo lattice model and not an artifact of DMFT.

In conclusion, we have clarified the physics behind the ferromagnetic metallic phase realized in the Kondo lattice model. We have demonstrated that the cooperation of ferromagnetism and partial Kondo screening results in an intriguing phase, here named spin-selective Kondo insulator, where an insulating state is stabilized for the minorityspin electrons while the majority-spin electrons are still metallic. We believe that the mechanism proposed here, the dynamically generated commensurability, should be generic for the ferromagnetic phase in the Kondo lattice models. It alternatively provides the nontrivial relation between the electron magnetization, spin polarization, and occupation number, for which the system can gain a maximum of additional energy. The proposed relation between the macroscopic quantities might be confirmed in experiments. Good candidates in this context are ferromagnetic heavy-fermion compounds, especially
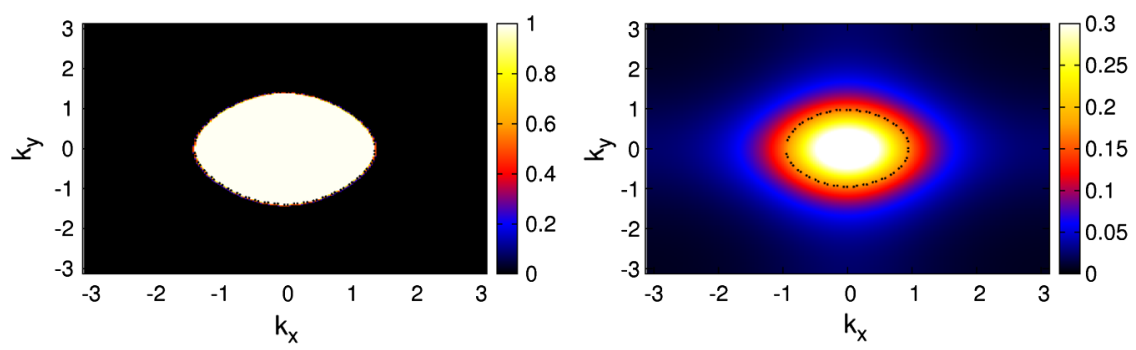

FIG. 5 (color online). Momentum-resolved occupation number $n(k)$ (same parameters as in Fig. 4). Left (right) panel shows the majority- (minority-) spin component. The dotted line represents the Fermi surface for noninteracting electrons (for the majority spin this line coincides with the shown surface). Note that for improving the contrast, the occupation for the minority-spin electrons is displayed in the interval $n(k) \in[0,0.3]$. 
compounds having a large Kondo temperature. For such compounds a verification of the above stated relation might be possible. Furthermore, spin-resolved transport measurements should show metallic majority-spin but insulating minority-spin electrons as well as a large Fermi surface for the minority-spin component.

We acknowledge fruitful discussions with A. Koga. R. P. thanks the Japan Society for the Promotion of Science (JSPS) and the Alexander von Humboldt Foundation. T. P. also gratefully acknowledges support by JSPS through the Bridge program. N.K. is supported by KAKENHI (No. 21540359, No. 20102008) and JSPS through its FIRST Program.

*peters@scphys.kyoto-u.ac.jp

[1] P. Coleman, in Handbook of Magnetism and Advanced Magnetic Materials, edited by $\mathrm{H}$. Kronmüller and S. Parkin (John Wiley \& Sons, New York, 2007), 1st ed., p. 95.

[2] P. Coleman and A. Schofield, Nature (London) 433, 226 (2005).

[3] P. Gegenwart and Q. Si, Nature Phys. 4, 186 (2008).

[4] S. Doniach, Physica (Amsterdam) 91B+C, 231 (1977).

[5] C. Krellner, S. Lausberg, A. Steppke, M. Brando, L. Pedrero, H. Pfau, S. Tencé, H. Rosner, F. Steglich, and C. Geibel, New J. Phys. 13, 103014 (2011).

[6] W. H. Lee, H. C. Ku, and R. N. Shelton, Phys. Rev. B 38, 11562 (1988).
[7] N. Perkins, J. Iglesias, M. Nunez-Regueiro, and B. Coqblin, Europhys. Lett. 79, 57006 (2007).

[8] S. Yamamoto and Q. Si, Proc. Natl. Acad. Sci. U.S.A. 107, 15704 (2010).

[9] G. B. Li, G. M. Zhang, and L. Yu, Phys. Rev. B 81, 094420 (2010).

[10] C. Lacroix and M. Cyrot, Phys. Rev. B 20, 1969 (1979).

[11] P. Fazekas and E. Muller-Hartmann, Z. Phys. B 85, 285 (1991).

[12] W. Metzner and D. Vollhardt, Phys. Rev. Lett. 62, 324 (1989).

[13] A. Georges, G. Kotliar, W. Krauth, and M. Rozenberg, Rev. Mod. Phys. 68, 13 (1996).

[14] T. Pruschke, M. Jarrell, and J. Freericks, Adv. Phys. 44, 187 (1995).

[15] K. Wilson, Rev. Mod. Phys. 47, 773 (1975).

[16] R. Bulla, T. Costi, and T. Pruschke, Rev. Mod. Phys. 80, 395 (2008).

[17] R. Peters, T. Pruschke, and F. B. Anders, Phys. Rev. B 74, 245114 (2006).

[18] A. Weichselbaum and J. von Delft, Phys. Rev. Lett. 99, 076402 (2007).

[19] R. Peters and T. Pruschke, Phys. Rev. B 76, 245101 (2007).

[20] J. Otsuki, H. Kusunose, and Y. Kuramoto, J. Phys. Soc. Jpn. 78, 034719 (2009).

[21] D. V. Efremov, J. J. Betouras, and A. Chubukov, Phys. Rev. B 77, 220401(R) (2008).

[22] H. Tsunetsugu, M. Sigrist, and K. Ueda, Rev. Mod. Phys. 69, 809 (1997).

[23] S. Smerat, U. Schollwöck, I.P. McCulloch, and H. Schoeller, Phys. Rev. B 79, 235107 (2009). 